\title{
Analysis of Reflection Properties of High Power Microwave Propagation in Mixture-Atmosphere
}

\author{
Tao Tang, Cheng Liao, Qingmin Gao, Pengcheng Zhao
}

Institution of Electromagnetics, Southwest Jiaotong University, Chengdu, China

Email: ttang@foxmail.com, c.liao@home.swjtu.edu.cn, kaocaomin@163.com,zhaopengcheng19860@163.com

Received June $3^{\text {rd }}, 2010$; revised July $21^{\text {st }}, 2010$; accepted July $21^{\text {st }}, 2010$

\begin{abstract}
A simple theoretical modeling is made to describe the reflection features of the high power microwave (HPM) in the mixture-atmosphere. The time-space dependent mixture-atmosphere is generated by ionization of the neutral molecules in atmosphere. Reflection will occur when HPM propagates in such mixture-atmosphere. The reflection characteristic of the HPM propagation in the mixture-atmosphere is investigated by FDTD numerical experiments in inhomogeneous medium, the influence on the reflection for different HPM parameters is concluded. An additional stability conditions for the FDTD difference scheme of the HPM mixture-atmosphere propagation model are presented.
\end{abstract}

Keywords: High Power Microwave, Reflection Properties, Mixture-Atmosphere

\section{Introduction}

When high power microwave (HPM) pulse propagates in atmosphere, the breakdown will occur if the corresponding region where the breakdown threshold is lower than the HPM electric field intensity. The free electrons which exist in the atmosphere are accelerated, the impact ionization is expected to occur when those accelerated electrons interacts with the neutral molecules, this process generates new electrons, the new electrons will be accelerated and the impact ionization will occur again, engendering more electrons. Due to the chain ionization, the electron density in the atmosphere rise sharply in a short time and space-time dependent plasma is formed [1]. The plasma will have some negative impacts on the HPM, bringing some nonlinear effects, such as reflection, absorption attenuation and etc. Because of the pulse width is limited, and it will take some time for the electrons produced by ionization reach the density which can significantly absorb the pulse, but before that time, the leading edge of the pulse has passed, and therefore, the attenuation occurs only in the tail. This mechanism is called as "tail erosion" [1]. Some theoretical and experimental work has been reported in the past few years. Woo and DeGroot [2] obtained the kinetic and hydrodynamic description of this problem by reduced the Boltzmann equation. Ali [3] has investigated the breakdown parameters for very high values of E/p. Kuo and Zhang [4] conducted a chamber ex- periment and identified tow mechanisms responsible for tow different degree of tail erosion. Yee et al. $[5,6]$ developed one-dimensional codes to investigate the dynamic behavior of short-pulse by using electron fluid equations and Maxwell's equations self-consistently.

When air is breakdown, the density is so high that the atmosphere will be turned into the plasma and nonplasma mixture-atmosphere. HPM propagates in the mixture-atmosphere, the reflection will occur, if the reflection is strong, there will have a negative impact on the HPM source device, even damages like airborne high-power radar or other HPM equipments.

In this paper, we report the results of a theoretical and numerical study of HPM reflection properties. Taking into account of the differential thinking, we treat the mixture-atmosphere as a layered inhomogeneous medium. And using FDTD method [7] in inhomogeneous medium, we computationally investigate the reflection characteristics of the HPM pulse in mixture-atmosphere; the stability conditions for the FDTD difference scheme of the HPM atmosphere propagation model are derived.

The paper is organized as following. In Section 2, the HPM propagation model in mixture-atmosphere is introduced, and its stability condition is presented. Section 3 presents the stability condition for FDTD difference scheme. Section 4 discusses the reflection properties of HPM propagation in the mixture-atmosphere. Conclusions are drawn in Section 5. 


\section{Propagation Model in Inhomogeneous Medium}

\subsection{Transmission Line Model for Inhomogeneous Medium}

The mixture-atmosphere which is composited by plasma and non-plasma will be counterproductive in the HPM to make the occurrence of attenuation. The attenuation will weaken the ionization, therefore, the electron density decreases along the wave propagation direction, and the mixture-atmosphere is not uniform. Taking into account of the differential thinking, the mixture-atmosphere can be discretized as a multi-layer medium approximation model which distributes along the propagation direction. The setup of this model is shown in Figure 1(a). If the division of each layer is small enough, the medium within each layer can be regarded as homogeneous medium, the dielectric of each layer is a constant.

According to the transmission line model, the propagation of electromagnetic waves along the $\mathrm{z}$ axis can be equivalent to the cascaded transmission line. By making this equivalent, respectively the reflection, transmission and other law for electromagnetic wave propagation in the layered medium can be obtained [8]. The $k_{i}$ and $Z_{i}$ in Figure 1 is the wave vector and wave impedance.

\subsection{The FDTD Difference Scheme of HPM in Mixture-Atmosphere}

The HPM atmosphere propagation model consists of Maxwell's equations and the electron fluid equations which are reduced from the Boltzmann equation [4-6].

$$
\begin{gathered}
\nabla \times \mathbf{E}=-\frac{\partial \mathbf{B}}{\partial t} \\
\nabla \times \mathbf{H}=e n_{e} \mathbf{u}+\frac{\partial \mathbf{D}}{\partial t}
\end{gathered}
$$

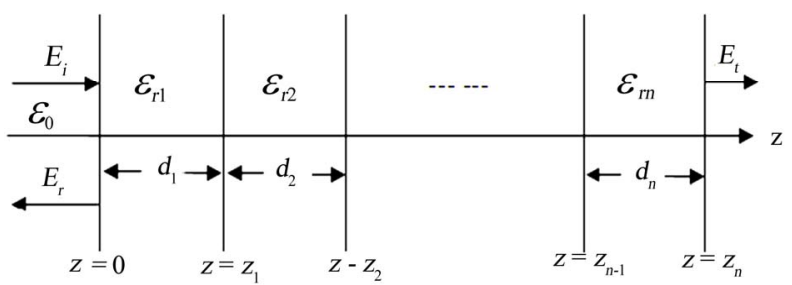

(a)

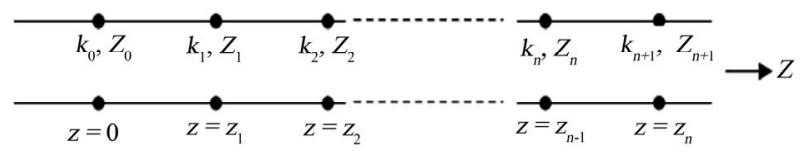

(b)

Figure 1. The schematic diagram of layered medium model for mixture-atmosphere (a) and equivalent circuit (b).

$$
\begin{gathered}
\frac{\partial n_{e}}{\partial t}=\left(v_{i}-v_{a}\right) n_{e} \\
m \frac{\partial\left(n_{e} \mathbf{u}\right)}{\partial t}=e n_{e} \mathbf{E}-n_{e} m v_{c} \mathbf{u}
\end{gathered}
$$

where (1) and (2) are the Maxwell's equations in the case of atmosphere. The avalanche ionization makes the number of free electrons increase rapidly. Equation (3) is the electron density continuity equation. Equation (4) is the electron momentum conservation equation in the fluid model. $\mathbf{E}$ is the electric field, $\mathbf{B}$ is the magnetic induction, $\mathbf{H}$ is the magnetic field, $\mathbf{D}$ is the displacement vector, $n_{e}$ and $\mathbf{u}$ is the density and velocity of electronic fluid, respectively; $v_{i}, v_{a}$ and $v_{c}$ are the ionization, attachment and collision rate coefficients; $e$ and $m$ denote the charge and mass of electronic, respectively.

In one dimensional model, the scalar forms of (1) and (2) in the inhomogeneous medium can be rewritten as

$$
\begin{gathered}
\frac{\partial E_{x}}{\partial t}=-v(z) Z(z) \frac{\partial H_{y}}{\partial z}-v(z) Z(z) e n u_{x} \\
\frac{\partial H_{y}}{\partial t}=-\frac{v(z)}{Z(z)} \frac{\partial E_{x}}{\partial z}
\end{gathered}
$$

In which $v(z)=1 / \sqrt{\varepsilon_{r} \mu}, Z(z)=\sqrt{\mu / \varepsilon_{r}}$ are the wave velocity and wave impedance of the medium, respectively. $\mu$ is the permeability and $\varepsilon_{r}$ is the relative permittivity of mixture-atmosphere, which can be modeled as [9].

$$
\sqrt{\varepsilon_{r}}=n_{n}-\frac{40.308 \times 10^{-12} n_{e}}{n_{n} f^{2}}
$$

where $n_{n}$ is the refraction index of non-ionizing atmosphere.

$$
n_{n}=1+\frac{77.6 \times 10^{-6}}{T}\left(p+\frac{4810 p_{e}}{T}\right)
$$

where $T$ is the atmosphere temperature in $\mathrm{K} ; \quad p$ and $p_{e}$ are pressure and water vapor pressure in $\mathrm{hPa}$, respectively; $f$ is the microwave frequency in $\mathrm{Hz}$; and $n_{e}$ is electron density in $\mathrm{m}^{-3}$. By discrete Equation (3), the changes of the electron density can be obtained; the related work can be found in [10]. The initial electron density is defined follows [11]

$$
n_{e 0}= \begin{cases}10 & (h<25 \mathrm{~km}) \\ 8 \times 10^{7} \times(h / 60)^{18} & (h \geq 25 \mathrm{~km})\end{cases}
$$

According to the Courant stability condition for the FDTD simulation, we have $v(z) \Delta t \leq \Delta z$ for the case of inhomogeneous medium. In order to meet this condition, the maximum wave velocity of the medium must be satisfied. Another stability condition must be met when use FDTD to solve the problem of HPM atmosphere propagation, which will be presented in the next Section. 


\section{Additional Stability Condition}

\subsection{Stability Condition}

The scalar equations of the (1)-(4) can be discretized by central difference method in the time step and spatial step. The FDTD difference scheme for these equations in one dimensional model can be written as

$$
\begin{gathered}
E_{x}^{n+1}(i)=E_{x}^{n}(i)-\frac{\Delta t}{\varepsilon_{r} \Delta z}\left[H_{y}^{n+\frac{1}{2}}\left(i+\frac{1}{2}\right)-H_{y}^{n+\frac{1}{2}}\left(i-\frac{1}{2}\right)\right] \\
+\frac{e \Delta t}{4 \varepsilon_{r}}\left[n_{e}^{n+1}(i)+n_{e}^{n}(i)\right] \cdot\left[u_{x}^{n+\frac{1}{2}}\left(i+\frac{1}{2}\right)+u_{x}^{n+\frac{1}{2}}\left(i-\frac{1}{2}\right)\right] \\
H_{y}^{n+\frac{1}{2}}\left(i+\frac{1}{2}\right)=H_{y}^{n-\frac{1}{2}}\left(i+\frac{1}{2}\right)-\frac{\Delta t}{\mu \Delta z}\left[E_{x}^{n}(i+1)-E_{x}^{n}(i)\right] \\
n_{e}^{n+1}(i)=\left[\left(1+\frac{\Delta t\left(v_{i}-v_{a}\right)}{2}\right) /\left(1-\frac{\Delta t\left(v_{i}-v_{a}\right)}{2}\right)\right] n_{e}^{n}(i) \\
u_{x}^{n+\frac{1}{2}}\left(i+\frac{1}{2}\right)=\left[\left(1-\frac{\Delta t v_{c}}{2}\right) /\left(1+\frac{\Delta t v_{c}}{2}\right)\right] u_{x}^{n-\frac{1}{2}}\left(i+\frac{1}{2}\right) \\
+\frac{e \Delta t}{m\left(2+\Delta t v_{c}\right)} \cdot\left[E_{x}^{n}(i+1)+E_{x}^{n}(i)\right]
\end{gathered}
$$

where the superscript $n$ is the time steep and $i$ is the space steep. When the air is breaking down, the electron density is so high that the HPM will be reflected and absorbed, the microwave will decay quickly in the mixture-atmosphere which leads to the role of ionization $v_{i}$ decreases quickly and the electron adhesion $v_{a}$ plays a dominant role. But $v_{a}$ is so small that the electron density remains almost unchanged. Therefore, equation (12) is stable.

In order to investigate the stability for the FDTD scheme of other equations, we expand the electromagnetic wave into plane wave spectrum, and we have

$$
\begin{gathered}
E_{x}^{n}(i)=E_{0} \exp \left[-j_{0}\left(i k_{z} \Delta z-n \omega \Delta t\right)\right] \\
H_{y}^{n+\frac{1}{2}}\left(i+\frac{1}{2}\right)=H_{0} \exp \left[-j_{0}\left(\left(i+\frac{1}{2}\right) k_{z} \Delta z-\left(n+\frac{1}{2}\right) \omega \Delta t\right)\right]
\end{gathered}
$$

Because the electron fluid velocity equation is only coupled with the electric field strength $\mathbf{E}$. Therefore, the electron fluid velocity equation becomes a first-order linear differential equation, and its solution can be written as follows

$$
u_{x}=\frac{e\left(j \omega+v_{c}\right)}{m} E_{x}
$$

Substituting (14) into (16), we have

$$
u_{x}^{n+\frac{1}{2}}\left(i+\frac{1}{2}\right)=u_{0} \exp \left[-j_{0}\left(\left(i+\frac{1}{2}\right) k_{z} \Delta z-\left(n+\frac{1}{2}\right) \omega \Delta t\right)\right]
$$

On the other hand, we can plug (14) and (15) into the (11), after simplified, we have

$$
\begin{aligned}
-\frac{\Delta t}{\mu \Delta z} E_{0}\left[\exp \left(-j_{0} k_{z} \Delta z\right)-1\right]=H_{0} \exp \left(-j_{0} \frac{1}{2} k_{z} \Delta z\right) \\
\cdot\left[\exp \left(j_{0} \frac{1}{2} \omega \Delta t\right)-\exp \left(-j_{0} \frac{1}{2} \omega \Delta t\right)\right]
\end{aligned}
$$

In (18), the term $\exp \left(-j_{0} k_{z} \Delta z\right)-1$ can be rewritten as follows

$$
\begin{aligned}
\exp \left(-j_{0} k_{z} \Delta z\right)-1 & =\exp \left(-j_{0} \frac{1}{2} k_{z} \Delta z\right) \\
\cdot & {\left[\exp \left(-j_{0} \frac{1}{2} k_{z} \Delta z\right)-\exp \left(j_{0} \frac{1}{2} k_{z} \Delta z\right)\right] }
\end{aligned}
$$

Combining (18) and (19), we can obtain

$$
H_{0}=\frac{\Delta t \sin \left(\frac{1}{2} k_{z} \Delta z\right)}{\mu_{0} \Delta z \sin \left(\frac{1}{2} \omega \Delta t\right)} E_{0}
$$

Similarly, combining (17) and (14), after tedious simplification, we can rewrite (13) as follows

$$
\begin{aligned}
& u_{0} \exp \left(-j_{0} \frac{1}{2} k_{z} \Delta z\right) \exp \left(j_{0} \frac{1}{2} \omega \Delta t\right) \\
& -u_{0} \exp \left(-j_{0} \frac{1}{2} k_{z} \Delta z\right)\left[\left(1-\frac{\Delta t v_{c}}{2}\right) /\left(1+\frac{\Delta t v_{c}}{2}\right)\right] \\
& \cdot \exp \left(-j_{0} \frac{1}{2} \omega \Delta t\right)=\frac{E_{0} e \Delta t}{m\left(2+\Delta t v_{c}\right)}\left\{\exp \left[-j_{0}\left(k_{z} \Delta z\right)\right]+1\right\}
\end{aligned}
$$

Taking the collision frequency $v_{c}$ into account, the time step should meet the following condition [12]

$$
\Delta t \leq 1 / 10 v_{c}
$$

Then we have

$$
\frac{1-\Delta t v_{c} / 2}{1+\Delta t v_{c} / 2} \approx 1
$$

At the same time, the term $\exp \left[-j_{0}\left(k_{z} \Delta z\right)\right]+1$ in (21) can be rewritten as follows

$$
\begin{aligned}
\exp \left[-j_{0}\left(k_{z} \Delta z\right)\right]+1 & =\exp \left(-j_{0} \frac{1}{2} k_{z} \Delta z\right) \\
\cdot & {\left[\exp \left(-j_{0} \frac{1}{2} k_{z} \Delta z\right)+\exp \left(j_{0} \frac{1}{2} k_{z} \Delta z\right)\right] }
\end{aligned}
$$


Therefore, considering (23) and (24), (21) can be further simplified as follow

$$
u_{0}=j \frac{e \Delta t \cos \left(\frac{1}{2} k_{z} \Delta z\right)}{m\left(2+v_{c} \Delta t\right) \sin \left(\frac{1}{2} \omega \Delta t\right)} E_{0}
$$

As discussed above, when the air is breakdown, the electron density will reach a maximum value $n_{\max }$, and then remains almost unchanged $[2,12,13]$. So we can take the place of $n_{e}^{n+1}$ and $n_{e}^{n}$ in (10) with $n_{\max }$. Combining (14), (15) and (17), after tedious simplification, we finally obtain from (11)

$$
\begin{aligned}
j \sin \left(\frac{1}{2} \omega \Delta t\right) E_{0} & =\frac{\Delta t}{\varepsilon_{r} \Delta z} \cdot j \sin \left(\frac{1}{2} k_{z} \Delta z\right) H_{0} \\
& +\frac{e n_{\max } \Delta t}{2 \varepsilon_{r}} \cos \left(\frac{1}{2} k_{z} \Delta z\right) u_{0}
\end{aligned}
$$

Substituting (20) and (25) into (26), we have

$$
\frac{\sin ^{2}\left(\frac{1}{2} \omega \Delta t\right)}{\Delta t^{2}}=\frac{\sin ^{2}\left(\frac{1}{2} k_{z} \Delta z\right)}{\varepsilon_{r} \mu \Delta z^{2}}+\frac{e^{2} n_{\max } \cos ^{2}\left(\frac{1}{2} k_{z} \Delta z\right)}{2 \varepsilon_{r} m\left(2+v_{c} \Delta t\right)}
$$

With the use of the propagation constant $k_{z}=\omega \sqrt{\varepsilon_{r} \mu}$ and (22), (27) can be written as

$$
\frac{\omega^{2}}{4}\left(\frac{\sin ^{2}\left(\frac{1}{2} \omega \Delta t\right)}{\left(\frac{1}{2} \omega \Delta t\right)^{2}}-\frac{\sin ^{2}\left(\frac{1}{2} k_{z} \Delta z\right)}{\left(\frac{1}{2} k_{z} \Delta z\right)^{2}}\right)=\frac{e^{2} n_{\max } \cos ^{2}\left(\frac{1}{2} k_{z} \Delta z\right)}{4 \varepsilon_{r} m}
$$

Follow the same derivation of the Courant condition, we can obtain

$$
\Delta t \leq \frac{1}{v(z)}\left(\frac{1}{\Delta z^{2}}+\frac{e^{2} \mu n_{\max }}{4 m}\right)^{-1 / 2}
$$

This stability condition is similar to the result given in Ref. [14].

\subsection{Calculation Results}

In order to illustrate our analysis, a numerical example is presented. We set the field intensity of the incident sinusoidal HPM source is $1 \times 10^{5} \mathrm{~V} / \mathrm{m}$, the center frequency is $1 \mathrm{GHz}$ and the pulse width is $50 \mathrm{~ns}$. It is transmitted vertically from the altitude of $35 \mathrm{~km}$; Figure 2 records its time domain waveform of different altitude and the corresponding electron density. In order for clear comparison, we shift the time when the wave pulse just reaches the corresponding position to zero. Obviously, our numerical results well agrees with the earlier literature results.

As the previous analysis, when the breakdown is oc
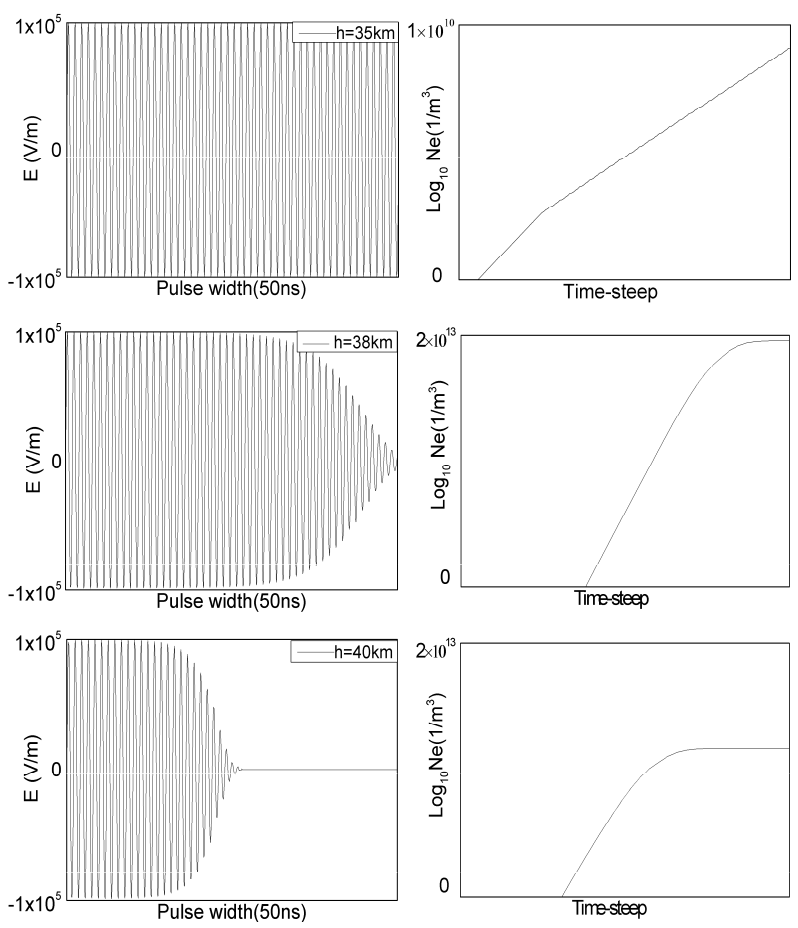

Figure 2. HPM decay in a mixture-atmosphere and the electron density of the corresponding position.

curring, the electron density grows exponentially in time quickly, the density is so high that the HPM will be reflected and absorpted, the microwave will decay quickly. This leads ionization decreases quickly and the electron adhesion plays a dominant role, therefore, the electron density will decreases slowly. Therefore, the use of FDTD in the case of inhomogeneous medium to deal with the problem of HPM atmosphere propagation is reasonable.

\section{Reflection in the Mixture-Atmosphere}

The air breakdown makes the atmosphere change into an inhomogeneous medium; HPM will be reflected on the surface of each layer of the mixture-atmosphere. In order to investigate the HPM reflection, the propagation area is divided into scattering field and the total field area, which shows in Figure 3.

According to the FDTD total field boundary conditions [15], adding a corresponding equivalent current at the total field outer boundary and a magnetic current at the total field boundary as incident wave of the tangential field components, while the normal component remains unchanged, that is

$$
\begin{aligned}
& H_{\mathrm{y}}{ }^{n+1 / 2}(\mathrm{i}-1 / 2)=H_{\mathrm{y}}{ }^{n-1 / 2}(\mathrm{i}-1 / 2) \\
& -\frac{c(\mathrm{z})}{Z(\mathrm{z})} \frac{\Delta t}{\Delta z}\left[E_{x}{ }^{n}(i)-E_{x}{ }^{n}(i-1)\right]+\frac{c(\mathrm{z})}{Z(z)} \frac{\Delta t}{\Delta z} E_{i}^{n}(i)
\end{aligned}
$$






Figure 3. Regional division: Scattering Fields.

$$
\begin{aligned}
& E_{x}^{n+1}(i)=E_{x}^{n}(i)-c(z) Z(z) \frac{\Delta t}{\Delta z}\left[H_{y}{ }^{n+1 / 2}(i+1 / 2)\right. \\
& \left.-H_{y}{ }^{n+1 / 2}(i-1 / 2)\right]-c(z) Z(z) e \Delta t U_{x}^{n+1 / 2}(i+1 / 2) \\
& \quad+c(z) Z(z) \frac{\Delta t}{\Delta z} H_{i}^{n+1 / 2}(i-1 / 2)
\end{aligned}
$$

Using the same source which is given in Section 3. The results are shown in Figure 4, since the breakdown degrees are different at different altitudes, the different breakdown will lead to different plasma concentrations in the mixture-atmosphere, resulting in different degrees of reflection. When the air is breakdown, the electron density $n_{e}=10^{8} n_{e 0}$ [3,11]. So, from (9) the initial electron density is about $4893.2 \mathrm{~m}^{-3}$ at $35 \mathrm{~km}$ altitude.

As Figure 4 (a) shows, the electron density is excited by HPM is about $3.6 \times 10^{11} \mathrm{~m}^{-3}$, which has not yet reached the breakdown density. Therefore, there is no breakdown occurred, almost no reflection. From Figure 4 (b), we can see that as the altitude increases the breakdown begin to happen, and the electron density increases sharply, leading to serious reflection. As Figure 4 (b) shows, the reflected field actually as high as $43 \%$ at the altitude of $38 \mathrm{~km}$, which would undoubtedly harm the high-power emitter device.

Because of different pulse field strength, different pulse width and different frequency correspond to different breakdown threshold, so, the HPM pulses with different parameters at the same altitude will cause different breakdown. Comparing with the Figure 5 (a), Figure 5 (b) and Figure 4 (c), we can see that at the same altitude of $40 \mathrm{~km}$, the field strength and pulse width increase would lead to an increase of reflection, but as Figure 5 (c) shows, at the same altitude of $40 \mathrm{~km}$ the reflection will decrease with the frequency increases. This is because in our concerned altitude range, the breakdown threshold will be decreases with the increases of field intensity and pulse width, but it will increases as the wave frequency increases [13].

\section{Conclusions}

A theoretical physical model describing the propagation of HPM pulse through the mixture-atmosphere is developed in the present work. We treat the mix
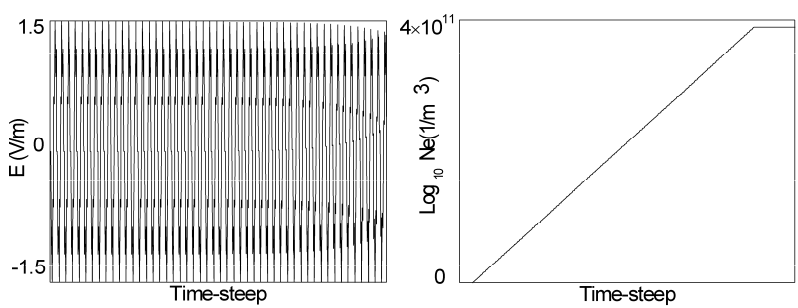

(a)

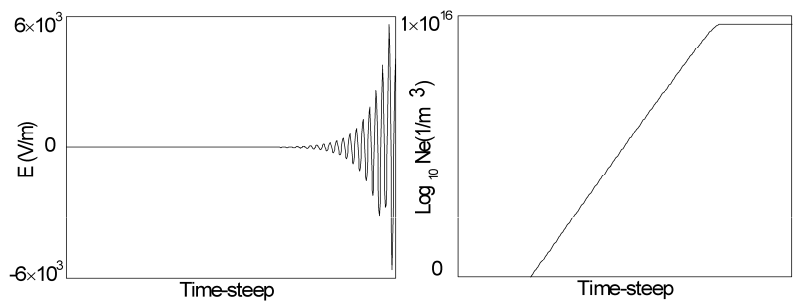

(b)

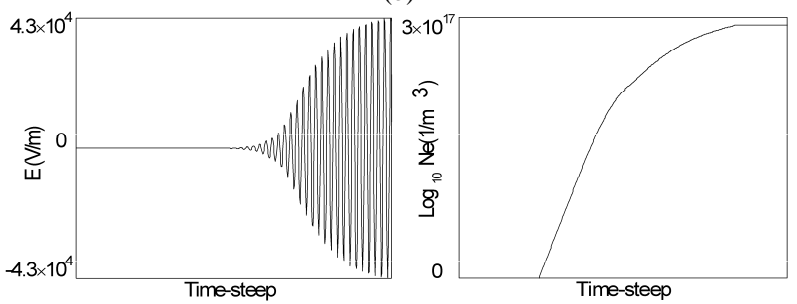

(c)

Figure 4. Reflection at different altitudes. (a) reflection field and electron density at $35 \mathrm{~km}$; (b) reflection field and electron density at $38 \mathrm{~km}$; (c) reflection field and electron density at $40 \mathrm{~km}$.

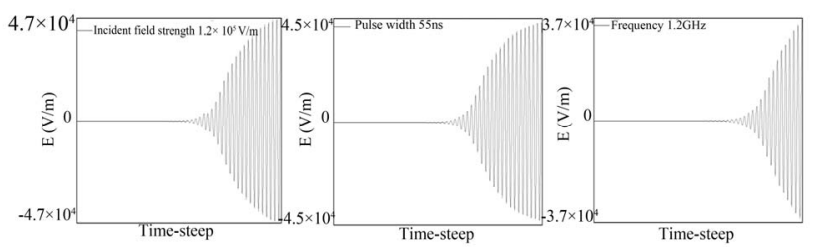

Figure 5. Reflection intensity of HPM sources with different parameters at $40 \mathrm{~km}$.

ture-atmosphere generated by HPM air breakdown as a layered inhomogeneous medium. Using the transmission line model of electromagnetic wave propagation in inhomogeneous medium and the FDTD method in inhomogeneous medium, the reflection characteristics of the HPM propagation in the mixture-atmosphere have been studied. The results show that the reflection increases with the degree of air breakdown. The stability conditions for the FDTD difference scheme of the HPM mixture-atmosphere propagation model are firstly presented.

\section{Acknowledgements}

Authors are very grateful to the Natural Science Foundation of China (60771020) for the financial assistance. 


\section{REFERENCES}

[1] S. P. Kuo, Y. S. Zhang and P. Kossey, "Propagation of High Power Microwave Pulses in Air Breakdown Environment," Physics Fluids, Vol. 133, No. 10, 1991, pp. 2906-2912.

[2] W. Woo and J. S. DeGroot, "Microwave Absorption and Plasma Heating Due to Microwave Breakdown in the Atmosphere,” Physics Fluids, Vol. 27, No. 2, 1984, pp. 475-487.

[3] A. W. Ali, "Nanosecond Air Breakdown Parameters for Electron and Microwave Beam Propagation,” Laser and Particle Beams, Vol. 6, No. 2, 1988, pp. 105-117.

[4] S. P. Kuo and Y. S. Zhang, "A Theoretical Model for Intense Microwave Pulse Propagation in an Air Breakdown Environment," Physics Fluids B, Vol. 3, No. 10, 1991, pp. 2906-2912.

[5] J. H. Yee, R. A. Alvarez, D. J. Mayhall, D. P. Byrne and J. DeGroot, "Theory of Intense Electromagnetic Pulses Propagation through the Atmosphere," Physics Fluids, Vol.29, No. 4, 1986, pp. 1238-1244.

[6] J. H. Yee, D. J. Mayhall, G. E. Sieger and R. A. Alvarez, "Propagation of Intense Microwave Pulses in Air and in a Waveguide," IEEE Transactions Antennas Propagation, Vol. 39, No. 9, 1991, pp. 1421-1426.

[7] A. Taflove and S. C. Hagness, "Computational Electrodynamics the Finite-Difference Time-Domain Method," 3rd edition, Reading, MA: Artech House, June 2005.

[8] H. S. Chen, Y. Wang and K. S. Chen, “Transient Analysis of Propagation in Non-uniform One Dimensional Media by Transmission Line Method," Journal of Microwaves,
Vol. 19, No. 3, 2003, pp. 25-29.

[9] J. Y. Wang and C. Y. Jiang, "Refractive Index of NonIonized and Ionized Mixture-Atmosphere,” Chinese Journal of Radio Science, Vol. 20, No. 1, 2005, pp. 34-36.

[10] T. Tang, C. Liao and D. Yang, "Feasibility Study of Solving High-Power Microwave Propagation in the Atmosphere Using FDTD Method," Chinese Journal of Radio Science, Vol. 25, No. 1, 2010, pp. 122-126.

[11] D. T. Hou, D. F. Zhou, Z. X. Niu and Z. Q. Yu, "Effect on Air Refraction Index by Effective Electric-Field Intensity in High Power Microwave Propagation," High Power Laser And Particle Beams, Vol. 16, No. 9, 2004, pp. 1183 -1185 .

[12] C. Zhang, D. F. Zhou, Y. P. Rao, Y. Chen and D. T. Hou, "FDTD Computation of Air Ionization and Breakdown Caused by High Power Microwave," High Power Laser and Particle Beams, Vol. 21, No. 5, 2009, pp. 719-723.

[13] M. Löfgren, D. Anderson and M. Lisak and L. Lundgren, "Breakdown-Induced Distortion of High-Power Microwave Pubes in Air," Physics Fluids B, Vol. 3, No. 12, 1991, pp. 3528-3531.

[14] M. Thèvenot, J. P. Bérenger, T. Monedière and F. Jecko, "A FDTD Scheme for the Computation of VLF-LF Propagation in the Anisotropic Earth-ionosphere Waveguide," Annals of Telecommunications, Vol. 54, No. 5-6, 1999, pp. 297-310.

[15] K. R. Umashanker and A. Taflove, "A Novel Method of Analyzing Electromagnetic Scattering of Complex Objects,” IEEE Transactions Electromagnetic Compatibility, Vol. EMC-24, No. 4, 1982, pp. 397-405. 